\title{
Estudiantes con discapacidad en la Universidad. Un estudio sobre su inclusión
}

\section{University students with disabilities in Universities. A study of their inclusion}

\author{
Alejandro RODRÍGUEZ-MARTÍN y Emilio ÁLVAREZ-ARREGUI \\ Universidad de Oviedo
}

Recibido: Abril 2013

Aceptado: Febrero 2014

\begin{abstract}
Resumen
El acceso de estudiantes con discapacidad a la Educación Superior se ha ido incrementando y las universidades están adoptando diferentes alternativas que deben ser valoradas. El objetivo de este estudio fue conocer la situación de una muestra de estudiantes con discapacidad $(\mathrm{n}=91)$ de una universidad española a través del diseño y validación de la Escala "CUNIDIS-d" de propiedades psicométricas satisfactorias. Los resultados avalan la importancia de realizar adaptaciones curriculares fundamentadas; adecuar la formación del profesorado; mejorar la accesibilidad e implicar al conjunto de la comunidad universitaria. Se concluye con distintas propuestas que respaldan la dimensión social del EEES.
\end{abstract}

Palabas clave: universidad; discapacidad; adaptaciones curriculares; estudiante.

\begin{abstract}
Access to higher education has increased among students with disabilities, and universities are adopting different alternatives which must be assessed. The purpose of this study was to identify the situation of a sample of students with disabilities $(n=91)$ who attend a university in Spain, through the design and validation of the "CUNIDIS-d" scale, with satisfactory psychometric properties. The results show the importance of making reasoned curriculum adaptations, adapting teacher training programmes, improving accessibility and involving all the university community. Different proposals were provided which support the social dimension of the EHEA.
\end{abstract}

Keywords: university; disability; academic accommodations; student.

Avanzamos hacia la plena implantación de un Espacio Europeo de Educación Superior (EEES) que ha definido un sistema universitario comparable y compatible en sus estructuras y titulaciones, orientando las universidades hacia la excelencia para convertirlas en el desarrollo socioeconómico de sus respectivas regiones. Sin embargo, desde los principios de la educación inclusiva, tal y como han destacado distintos autores (Ainscow, 2002; Allan y Slee, 2008; Barrio de la Puente, 2009) no es posible 
considerar una educación de calidad si no se flexibilizan los procesos de formación poniendo en valor el principio de igualdad de oportunidades. La universidad no es ajena a esta consideración por lo que debe profundizarse en la dimensión social del EEES lo que hace necesario incorporar en los estándares que definen la excelencia de éstas instituciones su capacidad de adaptación para dar respuestas a nuevas realidades de las que queremos enfatizar en este artículo el progresivo aumento de estudiantes con discapacidad.

Las iniciativas de las instituciones de educación superior hacia este colectivo, tal y como afirma Salvador (2003), tienen un triple compromiso, a saber, la generación de un conocimiento crítico, el desarrollo profesional y la intervención social. De ahí la necesidad de aproximarnos a la situación de los estudiantes con discapacidad, profundizando en la influencia que los avances legales vienen suscitando en las universidades españolas al incorporar en sus estatutos o reglamentos de desarrollo la dimensión de la discapacidad.

\section{El acceso a los estudios universitarios: apuntes sobre la situación en Europa}

Las universidades españolas, al igual que sus homólogas europeas, han experimentado en los últimos años un incremento de los estudiantes con discapacidad que acceden a la Educación Superior. En España, la población universitaria con discapacidad es reducida (1\%) aunque este porcentaje se incrementa para aquellos estudiantes con discapacidad que quieren acceder a la Educación Superior y es aquí donde nos encontramos un mayor número de obstáculos (Peralta y Morales, 2007).

A este respecto es importante destacar que las diferencias entre los países europeos son notables puesto que si bien la igualdad de oportunidades se recoge como un principio clave del orden legal, su puesta en práctica es compleja como queda reflejado en las indicaciones que se ofrecen en la Guía sobre la accesibilidad en la educación superior (David, 2004). Las cifras sobre estudiantes con discapacidad recogidas en diversos estudios realizados a lo largo de la última década (EADSNE, 2011, Eurydice, 2012; OCDE, 2003; Orr, Gwosc y Netz, 2011) constatan este incremento pero también que debería ser aún más significativo (Ebersold, 2008; Watkins, 2011). En esta línea, el Informe EuroStudent de 2012 recoge que el $11 \%$ de los estudiantes participantes manifestaron algún tipo de discapacidad que repercutía en sus estudios universitarios. Estos datos modifican al alza la tendencia si bien las informaciones estadísticas deben tomarse con cautela ya que son muchas las disonancias existentes entre las declaraciones voluntarias que realizan los estudiantes sobre su discapacidad y la situación real de las universidades (Meijer, Soriano y Watkins 2006).

Así, en otro estudio comparado (Escandell y Rodríguez-Martín, 2008) donde se analizó la legislación universitaria sobre discapacidad en los países de la Unión Europea, se concluyó que los cambios normativos referidos a estas cuestiones perseguían un doble objetivo, por un lado, la mejora de los derechos de los estudiantes $\mathrm{y}$, por otro, integrarlos en la misión de las universidades buscando equilibrios con el compromiso social asumido por estas instituciones. A este respecto, en el ámbito 
europeo, destacan entre otras leyes que incorporan cuestiones concretas sobre discapacidad y universidad, The Disability Discrimination Act-DDA (1995) de Reino Unido y reemplazada en 2010 por The Equality Act; la Loi pour l'égalité des droits et des chances des personnes handicapées de Francia (2005) o la Legge relativa all'assistenza l'integrazione sociale e i diritri delle persone handicappare (1992) de Italia a la que debemos añadir la Lag om likabehandling av studenter $i$ högskolan (2001) de Suecia, quizás, la norma más avanzada para afrontar la discriminación en la Universidad.

También debe destacarse que esta legislación se ha visto influenciada por otro conjunto de resoluciones promovidas por Naciones Unidas, lo que ha permitido construir un marco de referencia del que extraer principios sólidos de actuación. Así, entre los principales hitos destacan la Declaración Mundial sobre educación para todos (1990), la Conferencia Mundial sobre Educación Superior (1998) y la Conferencia Mundial sobre Necesidades Educativas Especiales (1994).

La Unión Europea también ha impulsando un conjunto de iniciativas en materia de discapacidad (Horizon, Helios I y II con su famosa Carta de Luxemburgo, eEurope, eQual, eInclusion, 2010-Una Europa Accesible) que han contribuido a la aprobación en 2000 de la Carta de los Derechos Fundamentales de la Unión Europea y la Comunicación de la Comisión Europea Hacia una Europa sin barreras para las personas con discapacidad que establece el principio de transversalidad 0 Mainstreaming disability (European Disability Forum, 2007; Naciones Unidas, 2009; Priestley, 2008). Sin embargo, el compromiso de mayor alcance promovido por Naciones Unidas ha sido, desde nuestro punto de vista, la aprobación de la Convención sobre los Derechos de las Personas con Discapacidad (2006) donde se recoge explícitamente que "los Estados Partes asegurarán que las personas con discapacidad tengan acceso general a la educación superior (art. 24)".

La evolución legal de nuestro país no ha sido ajena a estos cambios y desde la LISMI (1982), la derogada LRU (1983) y las sucesivas leyes reguladoras del sistema educativo, hemos avanzado hasta la Ley 51/2003, de 2 de diciembre, de igualdad de oportunidades, no discriminación y accesibilidad universal de las personas con discapacidad-LIONDAU donde se pone en valor el principio de transversalidad de las políticas y la mejora de la calidad de vida como reto fundamental.

No obstante, el punto de inflexión para los estudiantes con discapacidad en la universidad se sitúa en la Ley Orgánica 6/2001, de 21 de diciembre de UniversidadesLOU y, posteriormente, en la Ley Orgánica 4/2007, de 12 de abril que la modifica (LOMLOU). Este marco legislativo incluye la obligación de prestar atención a las personas con discapacidad por parte de administraciones y universidades, garantizando su acceso y permanencia en los estudios universitarios (art. 45). Además, plantea cambios sustanciales como la accesibilidad total de edificios, instalaciones y servicios; la inclusión de aspectos formativos sobre discapacidad en las titulaciones universitarias; y la concepción de las personas con discapacidad como integrantes plenos de la comunidad universitaria (disposición adicional 24 ). 
En este contexto, el desarrollo del Espacio Europeo de Educación Superior proporciona un nuevo respaldo a las iniciativas anteriores al proponer una adecuación estructural y metodológica que suponen un cambio cultural de hondo calado que ha generado incertidumbre porque establece nuevos perfiles profesionales y funcionales para los docentes (Allan, 2011; García, Guerra, González, y Álvarez-Arregui, 2010) y para los estudiantes (Danermak 2009; García, 2006; Konur, 2006). Por ello, la dimensión social del EEES se viene comprometiendo con este proceso de cambio desde la primera Declaración de Berlín hasta la última en Budapest/Viena (2010) desde lo apoyos que proporciona a las universidades para que desplieguen entornos de formación accesibles para todos.

\section{La dimensión de la discapacidad en la universidad española}

Tras la aprobación de la LOU se inició un importante proceso de reforma del sistema universitario español que, si bien no contó con el alcance debido en materia de discapacidad, ha supuesto un paso adelante en el reconocimiento legal de esta realidad. Un ejemplo palpable lo encontramos en la LOU ya que sus referencias sobre a la discapacidad fueron incorporados en la práctica totalidad de las instituciones de educación superior en sus cartas fundamentales (Rodríguez-Martín, 2008) aunque también es cierto que su puesta en práctica ha sido desigual en cuanto al enfoque, alcance y medidas concretas que implicaba.

La LOMLOU supuso un nuevo impulso a la situación fruto de las reivindicaciones del movimiento asociativo que, como el CERMI, ha reiterado que la atención integral de los estudiantes con discapacidad en la universidad debe constituirse como un criterio preferente de calidad de la Educación Superior. Este tejido asociativo también ha considerado la necesidad de promover planes de acción integrales en la universidad para atender a la discapacidad (Cayo, 2008), desarrollar iniciativas globales de formación del profesorado, impulsar la realización de adaptaciones curriculares y establecer indicadores de calidad sobre los servicios de atención a las personas con discapacidad acordes con los establecidos por la Association on Higher Education and Disability (Díez, Verdugo, Campo, Alonso, Moral y Calvo, 2008).

Atendiendo a estas cuestiones parece lógico comprobar en qué medida las universidades recogen en sus estatutos la dimensión de la discapacidad en sus misiones y estrategias así como en la defensa y promoción que hacen de la igualdad de oportunidades, el derecho a la no discriminación y la mejora de la accesibilidad. Es reseñable, en este punto, que únicamente nueve universidades hacen referencia a la discapacidad, más allá de los estudiantes, incorporando acciones para el personal docente, investigador, de administración y de servicios. Algunas universidades manifiestan su intención de establecer políticas de ayudas y becas específicas para el colectivo de estudiantes con discapacidad pero son prácticamente inexistentes las referencias estatuarias o reglamentarias en cuanto a las adaptaciones curriculares aunque sí han proliferando guías de apoyo y asesoramiento así como recomendaciones y buenas prácticas en este sentido. 
Los estudios sobre la discapacidad en la Educación Superior no son tan abundantes, a diferencia de lo que ocurre en las etapas previas, si bien hay investigaciones destacadas que se han centrado en el acceso a los estudios universitarios (Castro y Abad, 2009; De la Red, de la Puente, Gómez y Carro, 2002; Nava-Caballero, 2012); los modelos de gobernanza (Danermak, 2009); las actitudes hacia los estudiantes universitarios con discapacidad (Alcantud, Ávila y Asensi, 2000; Bilbao, Martínez, De Juan y García, 2012; Sachs y Schreuer, 2012), los servicios y programas de apoyo universitario (Álvarez, Alegreo y López, 2012; Díez et al, 2008; Forteza y Ortego, 2003; Trujillo y Cayo, 2006), la actitud del profesorado (Avramidis y Norwich, 2002; Fernández Batanero, 2011), la accesibilidad y el diseño universal (Fernández, Álvarez y Malvar, 2012; Guasch, Dotras y Llinares, 2010). Sin embargo, son menos frecuentes las investigaciones que hayan dado voz al propio colectivo de estudiantes con discapacidad y que, al igual que los estudios sobre actitudes, constituyen un aspecto determinante para adecuar las iniciativas institucionales, impulsar programas de formación y promover mejoras.

\section{Método}

En este trabajo se presentan los resultados obtenidos mediante el instrumento CUNIDIS-d (Rodríguez-Martín y Álvarez-Arregui, 2013) en un estudio de carácter descriptivo-exploratorio que forma parte de una investigación más amplia centrada en el análisis comparado de la situación de los estudiantes con discapacidad en diferentes universidades españolas y portuguesas.

\section{Objetivos}

El objeto del presente estudio es conocer las percepciones que sobre su propia situación tienen los estudiantes con discapacidad de todas las ramas de conocimiento. De forma concreta se pretendió indagar las opiniones de este colectivo hacia cuatro dimensiones clave: la realización de adaptaciones curriculares, la accesibilidad, la actitud del profesorado y la relación que mantienen con el conjunto de la comunidad universitaria. También se ha tratado de validar el cuestionario CUNIDIS-d (versión adaptada).

\section{Participantes}

La muestra estuvo constituida por 91 estudiantes con discapacidad que suponen el $69 \%$ de aquellos que voluntariamente han declarado su discapacidad durante el proceso de matrícula en una universidad canaria. La muestra se ha establecido siguiendo un método no probabilístico de conveniencia pues se pretendía seleccionar para el análisis el conjunto global de estudiantes con discapacidad, si bien quedó conformada por los que voluntariamente aceptaron participar en el estudio.

En su distribución, que sigue la tendencia de la población, contamos con $56.3 \%$ hombres y el $42 \%$ de mujeres con edades comprendidas entre los 22 y 28 años, lo que 
supone el $57.3 \%$ del porcentaje acumulado total de la muestra. En cuanto al tipo de discapacidad, el $49 \%$ es visual, el $37.5 \%$ física y el $15 \%$ auditiva lo que confiere, nuevamente, representatividad a la muestra en función del total de la población.

\section{Instrumento y procedimiento}

Se diseñó específicamente un instrumento denominado "CUNIDIS-D Cuestionario sobre Universidad y Discapacidad (Versión adaptada)" de acuerdo a las fases implicadas en el desarrollo de escalas de medida (Spector, 1992), la selección empírica de ítems (Prat y Doval, 2003) y una adecuada distribución y claridad en la evaluación (Vallejo, 2006).

Con la intención de asegurar la validez de contenido y la aplicabilidad del instrumento, en su primera versión, fue sometido al juicio de cinco expertos que analizaron la adecuación de los ítems con la dimensión que evaluaban, empleando el acuerdo inter-jueces para la eliminación de los ítems que generaban confusión. De esta forma, de los 89 ítems iniciales, propuestos tras la revisión de la literatura el instrumento se conforma con 40 ítems en su versión definitiva (Anexo) con una opción de respuesta que sigue el método Lickert con cinco posibles puntuaciones. Los jueces también indicaron la importancia de contemplar algunas preguntas abiertas que permitiera a los estudiantes formular iniciativas así como expresar libremente sus opiniones o matizarlas.

El cuestionario consta de nueve variables de clasificación (género, edad, titulación, curso, tipo de discapacidad, experiencia académica previa, apoyo familiar, contacto con otras personas con discapacidad, influencia en la decisión de cursar estudios universitarios) a las que acompañan un conjunto de preguntas que nos permitieron profundizar en el perfil de la muestra (elección de la titulación, consejos a otros estudiantes con discapacidad, opciones de futuro, participación en actividades sociales, representación universitaria,...).

El análisis de fiabilidad del instrumento muestra un $\alpha$ de Cronbach de .921 y la existencia de cuatro escalas unifactoriales acordes con las dimensiones que lo componen: Adaptaciones curriculares $(\alpha=.898)$; Acción docente $(\alpha=.931)$; Accesibilidad $(\alpha=.877)$ y Comunidad universitaria $(\alpha=.805)$. Estos datos se corroboran en el método de las dos mitades y la fórmula de corrección de SpearmanBrown $(\alpha=.89)$.

Para obtener la validez se realizó una correlación entre ítems demostrando que en todos los casos la corrección es superior a 0,73. También se realizó un análisis factorial exploratorio y, con un índice KMO .91 y p $<.001$ en la prueba de esfericidad de Bartlett, se obtuvieron los cuatro factores indicados con autovalores $>1$ y que en conjunto explicaban el $87.29 \%$ de la varianza total. Con el análisis descriptivo de los obtenemos coeficientes de asimetría y curtosis próximos al valor cero y por debajo del valor 2.0 respectivamente tal y como recomiendan Bollen y Long (1994), lo que indica semejanza con la curva normal. Para comprobar la estructura factorial en la población general, se realizó un análisis factorial confirmatorio con el módulo AMOS del paquete estadístico SPSS 19.0 que puso de manifiesto el buen ajuste el modelo que se proponía: 
$\chi 2=897,029, \mathrm{p}<.000 ; \mathrm{CFI}=, 96 ; \mathrm{RMSEA}=.63$; de acuerdo a los criterios de diversos autores (Byrne, 2010; Hu y Bentler, 1999).

La realización del estudio contó con la autorización del Vicerrectorado de Estudiantes que proporcionó la información necesaria para contactar con los estudiantes y solicitar su participación. Con los que manifestaron su predisposición se acordaron encuentros puntuales, en función de sus preferencias horarias y disponibilidad, y así aplicar el instrumento de manera presencial. También se remitió por correo el instrumento a los estudiantes que lo solicitaron. En colaboración con la ONCE, de manera específica, se adaptó el cuestionario a los estudiantes con discapacidad visual.

\section{Análisis de datos}

Los análisis de datos fueron realizados mediante el paquete estadístico SPSS 19.0. Se realizó un análisis de fiabilidad y validez de constructo del instrumento. Además se realizó un análisis factorial exploratorio de componentes principales y rotación Varimax y confirmatorio con el fin de analizar la estructura factorial del instrumento. A continuación se realizó un análisis de estadísticos descriptivos, un análisis de diferencias de medias en función del género y ANOVA (rama de conocimiento a la que se adscriben las titulaciones que se cursan y apoyo familiar). Se realizó un análisis de validez predictiva a través de la Lambda de Wilks que se mostró significativa $(\Lambda=.61$; $\chi 2=65.18 ; \mathrm{p}<.003)$ tomando como referencia el grado de apoyo mostrado por la familia ante la decisión de continuar estudios universitarios.

\section{Resultados}

Los resultados obtenidos nos permiten identificar la muestra del estudio desde el punto de vista de las titulaciones y facultad a la que pertenecen los estudiantes. La distribución es similar entre el Grado de Derecho y los Grados de Maestro en Educación Primaria y Educación Infantil que aglutina el 47\% de la muestra. En cuanto a las ramas de conocimiento, la de Ciencias Sociales y Jurídicas concentran el 56\%, Arquitectura e Ingenierías el 18\%, Humanidades el 16\% y Ciencias de la Salud el 10\%. La mayoría de los estudiantes cursan $3^{\circ}$ de sus respectivas titulaciones. (48\%).

Tratando de profundizar en el perfil de los estudiantes, el $46 \%$ recuerda su etapa escolar en el colegio e instituto con alegría y momentos tristes y el $60 \%$ manifiesta que los estudios superiores constituyen la etapa donde más dificultades han encontrado para integrarse. Ante ello, el 55\% manifiesta que sus respectivas familias les brindaron apoyo ante la decisión de continuar estudios universitarios frente al $40 \%$ que encontró oposición. En este sentido un $25 \%$ de los participantes indicó en las preguntas abiertas que esta oposición se justificaba porque las familias entendían que existían otras alternativas de formación, más adaptadas a la discapacidad, para seguir formándose al margen de la universidad o tener mejor perspectivas laborales.

En esta misma línea, el $65 \%$ de los estudiantes manifiestan no tener otros familiares con discapacidad pero sí mantienen contacto habitual con otras personas con 
discapacidad, fundamentalmente con discapacidad visual (58.3\%), física (47.9\%) y auditiva $(27.1 \%)$, motivadas, fundamentalmente, por relaciones de ocio y amistad $(63 \%)$.

Con respecto a los servicios que ofrece la Universidad, los resultados muestran que los estudiantes valoran positivamente la accesibilidad de las clases (44\%), los lavabos (64\%), así como los pasillos y zonas comunes (63\%). Sin embargo consideran necesario mejorar este aspecto en los despachos del profesorado (62.7\%), las bibliotecas $(50 \%)$ y las salas de informática (39.6\%). Hay diversos servicios (comedores universitarios, residencias y zonas deportivas) donde la mayoría no ha expresado su opinión, un hecho destacable porque suponen espacios importantes para las relaciones sociales de este colectivo. En esta línea, el 85\% manifiesta no participar en actividades de ocio, tiempo libre o deportivas organizadas por la universidad ya que no son accesibles $(94 \%)$.

En cuanto al acceso a la universidad, el 97\% considera que la decisión de cursar estudios superiores fue personal aunque el $45.8 \%$ indica que la familia también influyó. En esta línea, el $96 \%$ afirma cursar los estudios que le gustan valorando sus propias capacidades como adecuadas para las titulaciones en las que están matriculados. Los participantes consideran que, si tuvieran que aconsejar a otros estudiantes con discapacidad a la hora de elegir una titulación, recomendarían elegir aquella que suscite mayor preferencia $(66.7 \%)$ pero teniendo en cuenta las salidas profesionales (63.6\%). El 73\% considera que tras finalizar en la universidad, la salida profesional principal será en el ingreso a la Administración Pública (72.9\%) y continuar formándose $(46 \%)$.

\section{Las adaptaciones curriculares}

Con respecto a la adaptación del currículo (Tabla 1), más del 72\% de los estudiantes con discapacidad consideran que el profesorado no debe adaptar ni los objetivos $(\underline{X}=1.83 ; \sigma=1.51)$, ni los contenidos $(X=1.72 ; \sigma=1.33)$ ni los criterios de evaluación $(\bar{X}=2.79 ; \sigma=1.51)$. Por el contrario, el $79 \%$ indica que el profesorado debe realizar adaptaciones en la metodología ( $\bar{X}=4.66 ; \sigma=.70)$, en los materiales empleados en las actividades $(\bar{X}=4.34 ; \sigma=.94)$ y el tiempo para la realización de exámenes y entrega de trabajos $(\bar{X}=4.32 ; \sigma=1.14)$. Se constata que los estudiantes con discapacidad consideran que las principales adaptaciones a realizar no deben realizarse en los elementos básicos del currículo sino que han de centrarse en aquellos aspectos que constituyen las adaptaciones de acceso. 


\begin{tabular}{|c|c|c|c|c|c|c|c|}
\hline \multirow{2}{*}{$\begin{array}{c}\text { Respecto a los estudiantes con discapacidad } \\
\text { el profesorado debe... }\end{array}$} & \multirow{2}{*}{$\bar{X}$} & & \multicolumn{5}{|c|}{ Resultados (\%) } \\
\hline & & & 1 & 2 & 3 & 4 & 5 \\
\hline 1. ...adaptar los objetivos de las asignaturas & 1.83 & 1.51 & 72.3 & 6.4 & 2.1 & 4.3 & 14.9 \\
\hline 2. ...adaptar los contenidos de las asignaturas & 1.72 & 1.33 & 72.3 & 6.4 & 6.4 & 6.4 & 8.5 \\
\hline $\begin{array}{l}\text { 3. ...adaptar la metodología empleada en las } \\
\text { clases }\end{array}$ & 4.66 & .70 & - & - & 12.8 & 8.5 & 78.7 \\
\hline 4. ...adaptar las actividades de las asignaturas & 4.00 & .98 & - & 6.4 & 27.7 & 25.5 & 40.4 \\
\hline $\begin{array}{l}\text { 5. ...adaptar los materiales empleados en las } \\
\text { actividades }\end{array}$ & 4.34 & .94 & - & 4.2 & 18.8 & 14.6 & 60.4 \\
\hline 6. ...adaptar los instrumentos y de evaluación & 4.21 & 0.97 & 72.4 & 4.7 & 13.6 & 26.3 & 55.4 \\
\hline 7. ...adaptar los criterios de evaluación & 1.79 & 1.61 & 42.2 & 23.4 & 12.8 & 6.4 & 13.8 \\
\hline 8. ...adaptar las prácticas de la carrera & 3.91 & 1.16 & 4.3 & 6.4 & 25.5 & 21.3 & 42.6 \\
\hline $\begin{array}{l}\text { 9. ...ampliar el tiempo para los exámenes y } \\
\text { entrega de trabajos }\end{array}$ & 4.32 & 1.14 & 6.4 & - & 14.9 & 12.8 & 66.0 \\
\hline 10. ...realizar tutorías de manera habitual & 4.66 & .67 & - & - & 10.6 & 12.8 & 76.6 \\
\hline
\end{tabular}

Tabla 1. Distribución de frecuencias en el factor "Adaptación en las asignaturas"

\section{La acción docente}

Al consultar sobre la acción docente (Tabla 2), los datos revelan que la mayoría de los estudiantes entienden que el profesorado no realiza ninguna adaptación curricular en la práctica cotidiana del aula. Al contrastar estos datos con los recabados anteriormente, observamos que los estudiantes mantienen la tendencia de respuesta negativa hacia las adaptaciones de objetivos, contenidos y evaluación. Sin embargo, el dato más destacable es que el $70 \%$ de los estudiantes consideran que tampoco se adapta la metodología $(\mathrm{X}=2.15 ; \sigma=1.15)$.

\begin{tabular}{|c|c|c|c|c|c|c|c|}
\hline \multirow{2}{*}{$\begin{array}{c}\text { Respecto a los estudiantes con discapacidad el } \\
\text { profesorado... }\end{array}$} & \multirow{2}{*}{$\bar{X}$} & \multirow{2}{*}{$\sigma$} & \multicolumn{5}{|c|}{ Resultados (\%) } \\
\hline & & & 1 & 2 & 3 & 4 & 5 \\
\hline 11. ... adapta los objetivos de las asignaturas & 1.40 & .97 & 78.7 & 12.8 & 2.1 & 2.1 & 4.3 \\
\hline 12. ....adapta los contenidos de las asignaturas & 1.40 & .97 & 78.7 & 12.8 & 2.1 & 2.1 & 4.3 \\
\hline 13. ....adapta la metodología empleada en las clases & 2.15 & 1.15 & 47.8 & 21.3 & 23.4 & 3.3 & 4.3 \\
\hline $\begin{array}{l}\text { 14. ... adapta las actividades a desarrollar en las } \\
\text { asignaturas }\end{array}$ & 2.49 & 1.23 & 27.7 & 23.4 & 27.7 & 14.9 & 6.4 \\
\hline $\begin{array}{l}\text { 15. ...adapta los materiales empleados en las } \\
\text { actividades }\end{array}$ & 2.51 & 1.23 & 25.5 & 27.7 & 23.4 & 17.0 & 6.4 \\
\hline 16. ....adapta los instrumentos de evaluación & 2.02 & 1.21 & 46.7 & 21.4 & 21.3 & 4.3 & 6.4 \\
\hline 17. ....adapta los criterios de evaluación & 1.37 & .98 & 71.3 & 16.6 & 2.6 & 3.1 & 6.4 \\
\hline 18. ....adapta las prácticas de la carrera & 2.23 & 1.27 & 34.0 & 25.5 & 21.3 & 10.6 & 6.4 \\
\hline $\begin{array}{l}\text { 19. ...amplía el tiempo para los exámenes y entrega } \\
\text { de trabajos }\end{array}$ & 2.64 & 1.34 & 21.3 & 34.0 & 19.1 & 10.6 & 14.9 \\
\hline 20. ...realiza tutorías de manera habitual & 2.89 & 1.49 & 21.3 & 19.1 & 19.1 & 19.1 & 19.1 \\
\hline
\end{tabular}

Tabla 2. Distribución de frecuencias en el factor "Acción docente" 


\section{La accesibilidad percibida}

En lo referido a la accesibilidad (Tabla 3), el $70.2 \%$ de estudiantes con discapacidad considera que la Facultad donde cursan sus estudios tiene barreras arquitectónicas $(\bar{X}=2.38 ; \sigma=1.28)$; el $57.4 \%$ manifiesta que el equipamiento de las clases no está adaptado a sus principales necesidades $(\mathrm{X}=2.62 ; \sigma=1.28)$ y el $71,6 \%$ opina que las condiciones de las aulas no favorecen el acceso y movilidad de los estudiantes $(\bar{X}=2.45 ; \sigma=1.18)$. Un dato importante es que el $74.4 \%$ considera que los materiales impresos y audiovisuales que habitualmente se emplea en las clases como material de trabajo y estudio no están adaptados $(\bar{X}=2.04 ; \sigma=.99)$ afirmando el $60 \%$ de los estudiantes que hay limitaciones para participar en las actividades desarrolladas en las clases $(\bar{X}=2.36 ; \sigma=1.55)$. Esta cuestión profundiza aún más en la línea apuntada por los estudiantes respecto a la falta de adaptaciones curriculares de acceso que debería hacer el profesorado para adaptar los procesos de enseñanza-aprendizaje a las características del alumnado (Alcantud, 2005)

De las cuestiones planteadas con respecto a la accesibilidad, los estudiantes encuestados sólo manifiestan un grado de acuerdo relativo (44.7\%) al entender que sí se emplean tecnologías para el seguimiento de las clases por parte de los estudiantes $(\bar{X}=3.09 ; \sigma=1.41)$.

\begin{tabular}{|c|c|c|c|c|c|c|c|}
\hline \multirow{2}{*}{$\begin{array}{c}\text { Respecto a la accesibilidad de } \\
\text { los estudiantes con discapacidad... }\end{array}$} & \multirow{2}{*}{$\bar{X}$} & \multirow{2}{*}{$\sigma$} & \multicolumn{5}{|c|}{ Resultados (\%) } \\
\hline & & & 1 & 2 & 3 & 4 & 5 \\
\hline $\begin{array}{l}\text { 21. ...la Facultad/Escuela no tiene barreras } \\
\text { arquitectónicas }\end{array}$ & 2.38 & 1.28 & 23.4 & 46.8 & 10.6 & 6.4 & 12.8 \\
\hline $\begin{array}{l}\text { 22. ...el equipamiento de las clases está } \\
\text { adaptado }\end{array}$ & 2.62 & 1.28 & 17.0 & 40.4 & 21.3 & 6.4 & 12.8 \\
\hline $\begin{array}{l}\text { 23. ...las condiciones de las clases favorecen el } \\
\text { acceso y movilidad }\end{array}$ & 2.45 & 1.18 & 17.0 & 54.6 & 7.1 & 12.8 & 8.5 \\
\hline $\begin{array}{l}\text { 24. ...la disposición de las clases permite el } \\
\text { trabajo en grupo }\end{array}$ & 2.79 & 1.33 & 18.8 & 27.1 & 22.9 & 14.6 & 14.6 \\
\hline $\begin{array}{l}\text { 25. ...se emplean tecnologías para el } \\
\text { seguimiento de las clases }\end{array}$ & 3.09 & 1.41 & 14.9 & 27.7 & 12.8 & 23.4 & 21.3 \\
\hline $\begin{array}{l}\text { 26. ...los materiales impresos / audiovisuales } \\
\text { están adaptado }\end{array}$ & 2.04 & .99 & 34.0 & 40.4 & 12.8 & 12.8 & - \\
\hline $\begin{array}{l}\text { 27. ...cuentan con apoyos humanos } \\
\text { materiales para seguir las clases }\end{array}$ & 2.17 & 1.01 & 29.8 & 36.2 & 21.3 & 12.8 & - \\
\hline 28. ...realizan todas las prácticas de la carrera & 3.34 & 1.45 & 4.3 & 14.9 & 23.4 & 25.5 & 25.5 \\
\hline $\begin{array}{l}\text { 29. ...participan en todas las actividades de } \\
\text { clase }\end{array}$ & 3.21 & 1.33 & 6.4 & 17.0 & 25.5 & 29.8 & 17.0 \\
\hline $\begin{array}{l}\text { 30. ...tienen similares dificultades que el resto } \\
\text { de los compañeros/as }\end{array}$ & 2.36 & 1.55 & 46.8 & 12.8 & 14.9 & 8.5 & 17.0 \\
\hline
\end{tabular}

Tabla 3. Distribución de frecuencias en el factor "Accesibilidad"

\section{La participación en la Comunidad universitaria}

Los resultados evidencian (Tabla 4) que el 70.2\% los participantes consideran que las campañas universitarias de concienciación sobre discapacidad no son adecuadas 
$(\overline{\mathrm{X}}=1.87 ; \sigma=1.05) \mathrm{y}$, en esa línea el mismo porcentaje de estudiantes manifiesta que el profesorado de la universidad no está formado para dar respuesta a este colectivo $(\mathrm{X}=2.19 ; \sigma=1.31)$ y que la propia universidad debe mejorar sus estructuras y enfoques sobre el aprendizaje para dar una respuesta coherente a este colectivo $(\mathrm{X}=2.49$; $\sigma=1.04)$.

Por otro lado, el $70.2 \%$ de los estudiantes indican que sus compañeros respetan la disposición de la clase facilitando así el su acceso y movilidad $(\overline{\mathrm{X}}=3.83 ; \sigma=1.15)$ aunque el $78.7 \%$ afirma que las relaciones con sus compañeros son buenas $(\bar{X}=4.09$; $\sigma=1.02$ ). Todos los encuestados consideran que un estudiante con discapacidad puede ser un buen profesional $(\overline{\mathrm{X}}=4.91, \sigma=.28)$.

\begin{tabular}{|c|c|c|c|c|c|c|c|}
\hline \multirow{2}{*}{$\begin{array}{c}\text { Respecto a la accesibilidad de } \\
\text { los estudiantes con discapacidad... }\end{array}$} & \multirow{2}{*}{$\overline{\mathrm{X}}$} & \multirow{2}{*}{$\sigma$} & \multicolumn{5}{|c|}{ Resultados (\%) } \\
\hline & & & 1 & 2 & 3 & 4 & 5 \\
\hline $\begin{array}{l}\text { 31. Las campañas de Universidad para la } \\
\text { sensibilización y concienciación sobre } \\
\text { discapacidad son adecuadas }\end{array}$ & 1.87 & 1.05 & 38.3 & 31.9 & 19.1 & 4.3 & 2.1 \\
\hline $\begin{array}{l}\text { 32. Todos los estudiantes pueden participar en } \\
\text { las actividades culturales, deportivas y de } \\
\text { ocio que organiza la Universidad }\end{array}$ & 2.19 & 1.31 & 29.8 & 25.5 & 19.1 & 14.9 & 4.3 \\
\hline $\begin{array}{l}\text { 33. Los compañeros respetan la disposición de } \\
\text { la clase para facilitar el acceso y movilidad } \\
\text { de estos estudiantes }\end{array}$ & 3.83 & 1.15 & 6.4 & 6.4 & 17.0 & 38.3 & 31.9 \\
\hline $\begin{array}{l}\text { 34. La comunicación con el profesorado es } \\
\text { fluida }\end{array}$ & 3.45 & 1.17 & 4.3 & 19.1 & 27.7 & 25.5 & 23.4 \\
\hline $\begin{array}{l}\text { 35. La relación de los estudiantes y el } \\
\text { profesorado de la Facultad/Escuela es } \\
\text { adecuada }\end{array}$ & 3.36 & 1.09 & 6.4 & 14.9 & 27.7 & 38.3 & 12.8 \\
\hline $\begin{array}{l}\text { 36. La relación de los estudiantes y el resto de } \\
\text { compañeros de la Facultad/Escuela es la } \\
\text { adecuada }\end{array}$ & 4.09 & 1.02 & 4.3 & 2.1 & 14.9 & 38.3 & 40.4 \\
\hline $\begin{array}{l}\text { 37. El profesorado de la Universidad está } \\
\text { formado para dar respuesta a los } \\
\text { estudiantes con discapacidad }\end{array}$ & 2.19 & .97 & 23.4 & 46.8 & 19.1 & 8.5 & 2.1 \\
\hline $\begin{array}{l}\text { 38. Un estudiante con discapacidad puede } \\
\text { estudiar cualquier carrera }\end{array}$ & 2.91 & 1.46 & 23.4 & 19.1 & 19.1 & 19.1 & 19.1 \\
\hline $\begin{array}{l}\text { 39. Un estudiante con discapacidad puede ser } \\
\text { un buen profesional }\end{array}$ & 4.91 & .28 & - & - & - & 8.5 & 91.5 \\
\hline $\begin{array}{l}\text { 40. La Universidad está preparada para formar } \\
\text { y atender a estudiantes con discapacidad }\end{array}$ & 2.49 & 1.04 & 12.8 & 49.9 & 19.1 & 14.9 & 4.3 \\
\hline
\end{tabular}

Tabla 4. Distribución de frecuencias en el factor "Comunidad universitaria"

A continuación se recogen las principales diferencias de medias obtenidas (Tabla 5) y dado que la consistencia interna del cuestionario y de las escalas que lo componen es alta, hemos estimamos conveniente mostrar el análisis tomando como referencia los valores absolutos de cada una de las escalas, destacando así las posibles diferencias significativas. La variable género no identificó ninguna diferencia significativa $\mathrm{p} \leq .05$. 


\begin{tabular}{|c|c|c|c|c|c|c|c|c|c|c|c|c|c|}
\hline \multirow{2}{*}{ Factor } & \multicolumn{2}{|c|}{ Área 1} & \multicolumn{2}{|c|}{ Área 2} & \multicolumn{2}{|c|}{ Área 3} & \multicolumn{2}{|c|}{ Área 4} & \multicolumn{2}{|c|}{ Área 5} & \multirow{2}{*}{$\mathbf{F}$} & \multirow{2}{*}{ Sig. } & \multirow{2}{*}{ Contraste } \\
\hline & $\overline{\mathrm{X}}$ & $\sigma$ & $\overline{\bar{X}}$ & $\sigma$ & $\overline{\mathrm{X}}$ & $\sigma$ & $\overline{\bar{X}}$ & $\sigma$ & $\overline{\mathrm{X}}$ & $\sigma$ & & & \\
\hline 1 & 32.00 & 7.26 & 30.29 & 7.21 & 29.76 & 7.52 & 31.65 & 6.70 & 29.30 & 6.76 & 13.96 & .000 & $\begin{array}{c}2,3<4 \\
4>3\end{array}$ \\
\hline 2 & 19.52 & 13.02 & 19.59 & 12.61 & 17.70 & 11.84 & 23.85 & 9.45 & 21.64 & 11.17 & 20.34 & .000 & $\begin{array}{c}3<4 \\
4>1,2,3,5\end{array}$ \\
\hline 3 & 24.90 & 8.26 & 22.01 & 7.59 & 24.03 & 7.65 & 26.13 & 7.01 & 24.23 & 7.56 & 15.40 & .000 & $4>2,3,5$ \\
\hline 4 & 29.50 & 7.37 & 28.34 & 7.55 & 27.21 & 8.81 & 32.12 & 6.41 & 28.29 & 8.11 & 39.66 & .000 & $4>1,2,3,5$ \\
\hline $\begin{array}{l}\text { Factor } \\
\text { Factor } \\
\text { Factor } \\
\text { Factor }\end{array}$ & $\begin{array}{l}\text { 1. Ada } \\
\text { 2. Acc } \\
\text { 3. Acc } \\
\text { 4. Con }\end{array}$ & $\begin{array}{l}\text { ptación } \\
\text { ón doc } \\
\text { esibilid } \\
\text { lunidad }\end{array}$ & $\begin{array}{l}\text { en las a } \\
\text { ente } \\
\text { ad } \\
\text { Univer }\end{array}$ & $\begin{array}{l}\text { asignat } \\
\text { sitaria }\end{array}$ & dras & & $\begin{array}{l}\text { Área } \\
\text { Área } \\
\text { Área } \\
\text { Área } \\
\text { Área }\end{array}$ & $\begin{array}{l}\text { Arte } \\
\text { Cier } \\
\text { Cier } \\
\text { Cier } \\
\text { Arq }\end{array}$ & $\begin{array}{l}\text { y Huma } \\
\text { cias; } \\
\text { cias de } \\
\text { cias Soc } \\
\text { iitectura }\end{array}$ & $\begin{array}{l}\text { nidade } \\
\text { a Saluc } \\
\text { iales y } \\
\text { e Inge }\end{array}$ & $\begin{array}{l}\text { s; } \\
\text { Jurídic } \\
\text { ierías }\end{array}$ & s; y & \\
\hline
\end{tabular}

Tabla 5. Diferencias significativas en función de la rama de conocimiento-ANOVA

Tomando como variable las áreas de conocimiento (Tabla 6) en las que se agrupan las diferentes titulaciones, encontramos que en todos los factores se registran diferencias que indican que los estudiantes de la rama de Ciencias Sociales y Jurídicas y la rama de Artes y Humanidades tienen puntuaciones superiores al resto y, de manera especial, destacan sobre los estudiantes de Ciencias de la Salud. La diferencias que se encuentran evidencian, al igual que otros estudios previos (Moreno, Rodríguez, Saldaña, y Aguilera, 2006; Polo, Fernández y Díaz, 2011) que la rama de conocimiento y las titulaciones que en ellas se adscriben pueden influir en las percepciones. Así, la visión que manifiestan los estudiantes puede estar influenciada por la realidad en la que actúan, muy diferente según la naturaleza de las titulaciones y ramas de conocimiento.

\begin{tabular}{|c|c|c|c|c|c|c|c|c|c|}
\hline \multirow{2}{*}{ Factor } & \multicolumn{2}{|c|}{ Apoyo } & \multicolumn{2}{|c|}{ Oposición } & \multicolumn{2}{|c|}{ Indiferencia } & \multirow{2}{*}{$\mathbf{F}$} & \multirow{2}{*}{ Sig. } & \multirow{2}{*}{ Contraste } \\
\hline & $\overline{\mathrm{X}}$ & $\sigma$ & $\overline{\mathrm{X}}$ & $\sigma$ & $\overline{\mathrm{X}}$ & $\sigma$ & & & \\
\hline 1 & 21.57 & 10.11 & 22.15 & 11.11 & 20.14 & 7.88 & 1.04 & .352 & $* * *$ \\
\hline 2 & 32.23 & 6.05 & 28.42 & 6.53 & 31.11 & 6.97 & 18.76 & .000 & $1>2,3$ \\
\hline 3 & 24.49 & 7.13 & 25.08 & 7.53 & 26.55 & 7.13 & 1.70 & .183 & $* * *$ \\
\hline 4 & 31.29 & 5.45 & 28.19 & 8.13 & 30.44 & 7.44 & 10.99 & .000 & $1>2,3$ \\
\hline \multicolumn{10}{|c|}{$* * *$ El contraste no encuentra diferencias entre los grupos con una $p \leq .05$} \\
\hline \multicolumn{10}{|c|}{$\begin{array}{l}\text { Factor 1. Adaptación en las asignaturas } \\
\text { Factor 2. Acción docente } \\
\text { Factor 3. Accesibilidad } \\
\text { Factor 4. Comunidad Universitaria }\end{array}$} \\
\hline
\end{tabular}

Tabla 6. Diferencias significativas en función del apoyo familiar-ANOVA 
El apoyo familiar brindado a los estudiantes con discapacidad ante su decisión de continuar los estudios universitarios se mostró como una variable predictiva que ha permitido agrupar a los estudiantes de acuerdo a su percepción. Así, los estudiantes que contaron inicialmente con el apoyo familiar muestran puntuaciones más altas vinculadas con la acción docente y las relaciones en la comunidad universitaria.

\section{Conclusiones}

Los datos obtenidos nos han permitido explorar el perfil de estudiantes con discapacidad que voluntariamente han participado en este estudio y que, en todo momento, expresaron el agradecimiento por darles voz ante su proceso de inclusión universitaria que tiene referentes claros en el ámbito normativo y que, sin embargo, no logra todos los resultados esperados en la práctica. También hemos podido recabar información de interlocutores válidos sobre su situación en la universidad y, de manera especial, en lo relativo a las adaptaciones curriculares y la participación, aspectos que están en la línea de otros estudios previos (Dalmau, Llinares, y Sala, 2011; Luque y Gutiérrez, 2014) que muestran la necesidad de que la acción docente contemple esta realidad.

Las adaptaciones curriculares en la universidad suscitan importantes controversias pues la educación superior tiene como fin el desarrollo de competencias profesionales concretas y no constituye una etapa obligatoria. A este respecto, las informaciones recogidas nos ofrecen la percepción negativa de los estudiantes hacia la adaptación de objetivos, contenidos y evaluación. Una posible explicación la encontramos en que se perciba que estas modificaciones puedan menoscabar la formación recibida o, incluso, que la normativa no lo ampare. Sin embargo, el referente de las competencias profesionales es el eje central de estas estrategias pues, mientras las competencias no se alteren y su consecución esté garantizada, las posibles adaptaciones de acceso al currículo (poco significativas) y de sus elementos básicos (significativas) son estrategias válidas en los estudios universitarios, tal y como apunta Arnaiz (2000).

En esta línea, de acuerdo con Alcantud, Ávila y Asensi (2000), entendemos que la elaboración de las adaptaciones curriculares en la universidad debería seguir el mismo planteamiento que se realiza en las etapas no universitarias, adoptando en su realización directa una dimensión institucional promovida por el Estatuto del Estudiante Universitario. Este proceso requiere, necesariamente, contemplar dos aspectos puestos de relevancia en la literatura (Allan, 2010; Forteza y Ortego, 2003; Shevlin, Kenny y McNeela, 2004; Wai y Man, 2006) sobre la formación del profesorado y la sensibilización de la comunidad universitaria encaminada a una participación plena de los estudiantes con discapacidad.

Los planes de formación deben contemplar al profesorado novel y al resto del PDI ya que el contacto, la formación y la información recibida se han mostrado como variables determinantes que influyen en las actitudes más positivas y en la mejora de las relaciones en la comunidad universitaria (Suriá, 2011). En cuanto los contenidos, tendrán que abordar la normativa de referencia; los servicios e iniciativas de los que dispone la universidad; los tipos de discapacidad más habituales y sus repercusiones en 
el aprendizaje, así como las necesidades más frecuentes. El aprendizaje basado en problemas y el estudio de casos deben estar presentes en cualquier diseño metodológico de estas acciones formativas ya que permiten al profesorado reflexionar ante las posibles adaptaciones curriculares compartiendo las inquietudes con otros colegas de la misma y distinta titulación. Para un mayor grado de profundización, se estima conveniente desarrollar una formación inicial de carácter general y, posteriormente, otra específica adaptada a la naturaleza de las áreas de conocimiento. Son destacables en esta línea las acciones de formación que el ICE de la Universidad de Oviedo viene realizando desde 2009 hasta la actualidad.

En cuanto a las limitaciones del estudio, la muestra es reducida y los datos no permiten generalizar ni el perfil ni las opiniones recogidas. Hubiera sido interesante complementar este estudio cuantitativo con otras metodologías cualitativas como los autoinformes o el desarrollo de entrevistas, así como contrastarlas con las opiniones del profesorado lo que, a buen seguro, permitirán matizar y profundizar en las opiniones recabadas. Finalmente, sería importante desarrollar futuras investigaciones con muestras más amplias y en contextos universitarios distintos que permitieran confirmar la importancia de las variables analizadas. Por su parte, una línea por explorar es el análisis del impacto en la práctica docente del profesorado participante en acciones de formación sobre discapacidad. Asimismo, serían adecuados el desarrollo de estudios longitudinales para conocer la evolución de los estudiantes con discapacidad en el contexto universitario, así como el estudio de tasas de éxito y deserción.

\section{Referencias bibliográficas}

AINSCOW, M. (2002). Rutas para el desarrollo de prácticas inclusivas en los Sistemas Educativos. Revista de Educación, 327, 69-82.

ALCANTUD, F. (2005). La integración de los alumnos con n.e.e. en los estudios superiores. En M. López y R. Carbonell (Coords.), La integración educativa y social. "Veinte años después de la LISMI" (pp. 137-162). Barcelona: Ariel.

ALCANTUD, F., ÁVILA, V. y ASENSI, C. (2000). La integración de estudiantes con discapacidad en los estudios superiores. Valencia: Servei de Publicacions de la Universitat de València Estudi General.

ALLAN, J. (2010). The sociology of disability and the struggle for inclusive education. British Journal of Sociology of Education, 31(5), 603-619.

ALLAN, J. (2011). Civilising teacher education? Learning with diversity. Teacher Education Practice, 24(3), 351-353.

ALLAN, J. y SLEE, R. (2008). Doing Inclusive Education Research. Rotterdam: Sense Publishers.

ÁlVAREZ, P.R., ALEGRE, O.M. y LÓPEZ, D. (2012). Las dificultades de adaptación a la enseñanza universitaria de los estudiantes con discapacidad: Un análisis desde un enfoque de orientación inclusiva. Relieve, 18(2), 1-18. 
ARNAIZ, P. (2000). Las adaptaciones en el curriculum universitario. Boletín del Real Patronato, 47, 151-168.

AVRAMIDIS, E. y NORWICH, B. (2002). Teachers'attitudes toward integration/inclusion: a review of the literature. European Journal of Special Needs Education, 17(2), 129-147.

BARRIO DE La PUENTE, J.L. (2009). Hacia una Educación Inclusiva para todos. Revista Complutense de Educación, 20(1), 13-31.

BILBAO, M.C., MARTÍNEZ, M.A., DE JUAN, M.N., y GARCÍA, M.I. (2012, noviembre). Evolución de los servicios de apoyo a personas con discapacidad en las universidades españolas. Ponencia presentada en el I Congreso Internacional Universidad y Discapacidad, Madrid, España.

BOLLEN, K.A. y LONG, J.S. (1994). Testing structural equation models. Newbury Park: Sage.

BYRNE, B.M. (2010). Structural equation modeling with Amos: Basic concepts, applications and programming. New Yorrk: Routledge.

CASTRO, J.F. y ABAD, M. (2009). La incorporación a los estudios superiores: situación del alumnado con discapacidad. Revista Qurriculum, 22, 165-188.

CAYO, L. (2008). Guía para la elaboración de un Plan de acción al alumnado con discapacidad en la universidad. La Cuestión Universitaria, 6, 103-116.

DALMAU, M., LlinARES, M., y Sala, I. (2011). Percepción de los estudiantes universitarios españoles con discapacidad sobre los apoyos recibidos en su formación universitaria. En J.M. Román, M.A. Martín, y J.D Valdivieso (Comp.). Educación, aprendizaje y desarrollo en una sociedad multicultural (pp. 60996111). Madrid: Ediciones de la Asociación Nacional de Psicología y Educación.

DANERMAK, B. (2009). Disabled Students um Hihger Education: Management for Inclusion. Higher Education Management, 11(3), 113-127.

DAVID, M.E. (2004). Equality and Equity in Higher Education: Learning to develop new paradigms from the US experience?. European Educational Research Journal, 3(4), 813-819.

DE LA RED, N., DE LA PUENTE, R., GÓMEZ, M.C., y CARRO, L. (2002). El acceso a los estudios superiores de las personas con discapacidad fisica $y$ sensorial. Valladolid: Secretariado de publicaciones de la Universidad de Valladolid.

DÍEZ, E., VERDUGO, M.A., CAMPO, M., SANCHO, I., ALONSO, A., MORAL, E., y CALVO, I. (2008). Protocolo de actuación para favorecer la equiparación de oportunidades de los estudiantes con discapacidad en la universidad. Salamanca: INICO.

EADSNE-European Agency for Development in Special Needs Education (2011). Mapping the Implementation of Policy for Inclusive Education: An exploration of 
challenges and opportunitiesfor developing indicators. Odense (Denmark): European Agency for Development in Special Needs Education.

EBERSOLD, S. (2008). Adapting higher education to the needs of disabled students: development, challenges and prospects in OECD. Higher education to 2030. Paris: OECD.

ESCANDELL, M.O. y RODRÍGUEZ-MARTÍN, A. (2008). Avances legales sobre diversidad en la universidad. International Journal of Developmental and Educational Psychology, 1, 137-147.

EUROPEAN DISABILITY FORUM (2007). Creating an inclusive society: Mainstreaming disability based on the Social Economy example. Brussels: Edition \& Imprimerie.

EURYDICE (2012). The European Higher Education Area in 2012: Bologna Process Implementation Report. Brussels: Education, Audiovisual and Culture Executive Agency.

FERNÁNDEZ, M.D., ÁlVAREZ, Q., y MALVAR, M.L. (2012). Accesibilidad e inclusión en el Espacio Europeo de Educación Superior: el caso de la Universidad de Santiago de Compostela. Aula Abierta, 40(3), 71-82.

FERNÁNDEZ BATANERO, J.M. (2011). Competencias docentes para la inclusión del alumnado universitario en el marco del Espacio Europeo de Educación Superior. Revista de Educación Inclusiva, 4(2), 137-147.

FORTEZA, D. y ORTEGO, J.L. (2003). Los servicios y programas de apoyo universitario para personas con discapacidad. Estándares de calidad, acción y evaluación. Revista de Educación Especial, 33, 9-26.

GARCÍA, M.R. (2006). Las competencias de los alumnos universitarios. Revista Interuniversitaria de Formación del Profesorado, 20(3), 253-269.

GARCÍA, M.R.; GUERRA, S.; GONZÁLEZ, N. y ÁLVAREZ ARREGUI, E. (2010). Estudio exploratorio de las percepciones del profesorado universitario respecto a la gestión de la docencia. Educación XX1, 13(2), 163-184.

GUASCH, D., DOTRAS, P., y LLINARES, M. (2010). Los principios de Accesibilidad Universal y Diseño para Todos en la Docencia Universitaria. Córdoba: Servicio de publicaciones de la Universidad de Córdoba.

HU, L. y BENTLER, P. (1999). Cutoff criteria for fit indexes in covariance structure analysis: Convectional criteria versus new alternatives. Structural Equation Modelin: A Multidisciplinary Journal, 6(1), 1-55.

KONUR, O. (2006). Teaching disabled students in higher education. Teaching in Higher Education, 211(3), 351-363.

LUQUE, A. y GUTIÉRREZ, R. (2014). La integración educativa y social del alumnado con discapacidad en el EEES: Universidad de Bolonia. Revista Complutense de Educación, 25(1), 153-175 
MEIJER, C.; SORIANO, V., y WATKINS, A. (2006). Educación Especial en Europa. Factores clave en la educación post-secundaria. Middelfart: European Agency for Development in Special Needs Education.

MORENO, J., RODRÍGUEZ, I.R., SALDAÑA, D., y AGUILERA, A. (2006). Actitudes ante la discapacidad en el alumnado universitario matriculado en materias afines. Revista Iberoamericana de Educación, 40(5), 1-7.

NACIONES UNIDAS (2009). Mainstreaming Disability in MDG Policies, Processes and Mechanisms: Development for All. Report of the Expert Group Meeting. New York: Secretariat for the Convention on the Rights of Persons with Disabilities Division for Social Policy and Development.

NAVA CABALLERO, E.Ma . (2012). El acceso y la integración de los estudiantes con discapacidad en la Universidad de León. Revista Complutense de Educación, 23(2), 293-316.

OECD (2003). Disability at higher education. Paris: OECD,

ORR, D., GWOSC, C., y NETZ, N. (2011). Social and Economic Conditions of Student Life in Europe. Synopsis of indicators. Final report. Eurostudent IV 20082011. Bielefeld: Bertelsmann Verlag.

PERALTA MORALES, A. (2007). Libro Blanco sobre universidad y discapacidad. Madrid: Real Patronato sobre Discapacidad y GRAFO S.A.

POLO, M.T., FERNÁNDEZ, C., y DÍAZ, C. (2011). Estudio de las actitudes de estudiantes de Ciencias Sociales y Psicología: Relevancia de la información y contacto con personas discapacitadas. Universitas Psychologica, 10(1), 113-123.

PRAT, R. y DOVAL, E. (2003). Construcción y análisis de escalas. En J.P. Lévy y J. Varela (Eds.). Análisis multivariable para las ciencias sociales (pp. 43-89). Madrid: Prentice Hall.

PRIESTLEY, M. (2008). Targeting and mainstreaming disability in the 2008-2012 National Strategy Reports for Social Protection and Social Inclusion. Leeds: Academic.

RODRÍGUEZ-MARTÍN, A. (2008, mayo). Universidad y diversidad ante el reto de la dimensión social del E.E.E.S. Ponencia presentada en el V Congreso Internacional de Psicología y Educación: Los retos del futuro. Oviedo, España.

RODRÍGUEZ-MARTIN, A. y ÁLVAREZ-ARREGUI, E. (2013). Development and validation of a Scale to identify attitudes towards disability in higher education. Psicothema, 25(3), 370-376.

SACHS, D. y SCHREUER, N. (2012). Inclusion of Students With Disabilities In Higher Education: Performance and Participation In Student's Experiences. Disability Studies Quarterly, 31(2), 1593-1561.

SALVADOR, F. (2003). Actitudes sociales ante la discapacidad. En R. Casado y A. Cifuentes (coords.). El acceso al empleo y a la universidad de las personas con 
discapacidad. Barreras y alternativas (pp. 19-34). Burgos: Servicio de Publicaciones de la Universidad de Burgos.

SHEVLIN, M., KENNY, M., y McNEELA, E. (2004). Participation in higher education for students with disabilities: an Irish perspective. Disability \& Society, 19(1), 15-30.

SPECTOR, P. E. (1992). Summated rating scale construction. Newbury Park: LEA.

SURIÁ, R. (2011). Análisis comparativo sobre las actitudes de los estudiantes hacia sus compañeros con discapacidad. Electronic Journal of Research in Educational Psychology, 9(1), 197-216.

TRUJILlO, E. y Cayo, L. (2006) (Dir). Guía de Recursos sobre Universidad y discapacidad. Madrid: Grupo Editorial Cinca.

VALLEGO, P. (2006). Medición de actitudes en Psicología y Educación. Madrid: Gráficas Ormag.

WAI, K. y MAN, D. (2006). Attitudes toward people with disabilities: A comparison between health care professionals and students. International Journal of Rehabilitation Research, 29(2), 155-160.

WATKINS, A. (2011) (Comp.). Special Needs Education Country Data 2010. Odense (Denmark): European Agency for Development in Special Needs Education.

\section{Correspondencia con los autores}

Alejandro RODRÍGUEZ MARTÍN

Emilio ÁLVAREZ ARREGUI

Facultad de Formación del Profesorado y Educación (Campus de Llamaquique)

$\mathrm{C} /$ Aniceto Sela $\mathrm{s} / \mathrm{n}$

33005 Oviedo (Asturias)

e-mail: rodriguezmalejandro@uniovi.es y alvarezemilio@uniovi.es 


\section{CUNIDIS-d \\ Cuestionario sobre UNIversidad y DIScapacidad}

Este cuestionario intenta recoger información sobre distintos aspectos de la vida universitaria de los estudiantes con discapacidad, para mejorar la accesibilidad, la información-formación y plantear las oportunas acciones de mejora.

Te pedimos total sinceridad en señalar aquello que desees, pues las respuestas son totalmente anónimas y confidenciales, siendo de uso exclusivo para este estudio. GRACIAS POR TU COLABORACIÓN.

\section{INFORMACIÓN GENERAL}

1. Género:

$\square$ Hombre $\square$ Mujer

2. Edad:

\begin{tabular}{|c|c|c|c|c|c|}
\hline$\square 18$ & $\square 19$ & $\square 20$ & $\square 21$ & $\square 22$ & $\square 23 \quad \square 24$ \\
\hline$\square 26$ & $\square 27$ & $\square 28$ & $\square 29$ & $\square 30$ & $\square$ Más de 30 \\
\hline
\end{tabular}

3. Titulación cursada actualmente:

4. Curso en el que estás matriculado o pertenecen la mayoría de tus asignaturas:
$\square 1^{\circ}$
$\square 2^{\circ}$
$\square 3^{\circ}$
$\square 4^{\circ}$
$\square 5^{\circ}$
$\square 6^{\circ}$

5. ¿En qué año comenzaste a estudiar en la Universidad?:

6. Tipo de discapacidad (señala las que procedan)
$\square$ Auditiva
$\square$ Física
$\square$ Visual
$\square$ Otras discapacidades y/o enfermedades

7. ¿Cómo recuerdas tu vida en el colegio y en el instituto?
$\square$ Con alegría
$\square$ Con alegría y momentos tristes
$\square$ Con tristeza e insatisfacción
$\square$ Con indiferencia
$\square$ Prefiero no pensar en ello
$\square$ Otras (Indicar cuáles):

8. ¿En qué nivel educativo has tenido más dificultades a la hora de tu integración?
$\square$ Educación Infantil/Educación Primaria
$\square$ Educación Secundaria Obligatoria
$\square$ Bachillerato/Formación Profesional
$\square$ Todos
$\square$ Ninguno

9. ¿Hay alguna otra persona con discapacidad en tu familia (señala las que procedan)?
$\square$ Mi padre
$\square$ Mi madre
$\square$ Alguno de mis hermanos
$\square$ Otros parientes (tíos, primos, abuelos, etc.)
$\square$ Ninguno 


\section{CUNIDIS- $d$}

10. Ante la decisión de continuar estudios universitarios, tu familia te ha mostrado...
$\square$ Apoyo
$\square$ Oposición
$\square$ Indiferencia

11. ¿Tienes o has tenido algún tipo de contacto con personas con discapacidad?

$\square$ Sí $\square$ No

12. ¿Cuál es la razón del contacto? (indicar las que procedan)
$\square$ Familiar
$\square$ Laboral
$\square$ Ocio/amistad
$\square$ En mi clase hay compañeros con discapacidad
$\square$ Voluntariado
$\square$ Otras razones (indicar cuáles)

13. ¿Con qué personas con discapacidad tienes o has tenido contacto?
$\square$ Personas con discapacidad visual
$\square$ Personas con discapacidad auditiva
$\square$ Personas con discapacidad física
$\square$ Personas con discapacidad psíquica
$\square$ Personas con otro tipo de discapacidad

14. ¿Conoces si la universidad dispone de un servicio de atención a los estudiantes con discapacidad? $\square$ Sí $\square$ No

15. Valora de 1 a 5 (donde 1 es la puntuación mínima y 5 la máxima) en qué grado son accesibles para las personas con discapacidad los servicios de la Universidad:
$\square$ Clases
Biblioteca
Pasillos
Cafetería
$\square$ Aparcamiento
Lavabos
Salones de actos
Laboratorios
Comedores
Salas de informática
Zonas deportivas
Despachos del profesorado
Residencias
Servicios Administrativos

\section{ESTUDIAR EN LA UNIVERSIDAD}

16. ¿Quién influyó en tu decisión de estudiar en la universidad?
$\square$ Tu familia
Tus amigos
Algún profesor/asesor

$\square$ Nadie
$\square$ Yo mismo

17. ¿Por qué escogiste la titulación que cursas?

$\square$ Por influencia de otros (padres, profesorado, orientadores, etc.)

Porque permite compatibilizarla con trabajo

Porque me gusta y tengo las capacidades necesarias

Por ser la más adecuada tras un proceso de asesoramiento

Porque mis amigos la habían elegido

Porque conocía alguna persona con discapacidad que la cursaba

Por las salidas profesionales que tenía

Otros (Indicar cuáles): 


\section{CUNIDIS-d}

18. ¿Qué aconsejarías a una persona con discapacidad a la hora de elegir una carrera?

$\square$ Que elija aquello que más le gusta

Que hable con personas con discapacidad

$\square$ Que tenga en cuenta las posibles salidas profesionales

Que pida asesoramiento en el Instituto

$\square$ Que pida asesoramiento en la Universidad

$\square$ Que no se complique la vida y que no siga el camino universitario

Otros (Indicar cuáles):

19. ¿Qué te planteas hacer cuando termines tu carrera?

$\square$ Opositar a la Administración Pública

$\square$ Montar mi propia empresa o negocio

$\square$ Buscar trabajo en empresas privadas

$\square$ Continuar mi formación a través de master y cursos de especialización

$\square$ Realizar otra carrera

Otros (Indicar cuáles):

20. ¿Participas en actividades de ocio, tiempo libre y deportivas dependientes u organizadas por tu Universidad?

$\square \mathrm{SI} \square \mathrm{NO}$

21. ¿Son estas actividades accesibles?

22. ¿Están destinadas a personas con discapacidad?

23. ¿Perteneces a alguna asociación universitaria?

24. ¿Has sido representante de estudiantes en la Universidad?

$\square$ SI $\square$ NO

\section{FORMACIÓN UNIVERSITARIA}

Te pedimos que expreses con sinceridad tu grado de acuerdo (de 1 a 5) con las afirmaciones que se realizan, referidas a los estudiantes con discapacidad, teniendo en cuenta que no existen respuestas buenas o malas, sino diferentes formas de pensar:

$$
\begin{array}{c|c|c|c|c}
\begin{array}{c}
1 \\
\text { Totalmente en } \\
\text { desacuerdo }
\end{array} & 2 & 3 & 4 & 5 \\
\text { Poco de acuerdo } & \text { De acuerdo } & \text { Muyde acuerdo } & \begin{array}{c}
\text { Totalmente de } \\
\text { acuerdo }
\end{array}
\end{array}
$$

\section{Adaptaciones curriculares}

Respecto a los estudiantes con discapacidad, el profesorado DEBE:

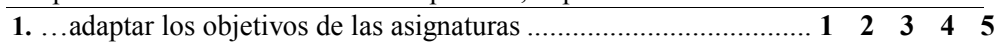

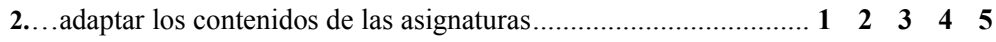

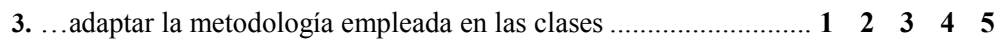

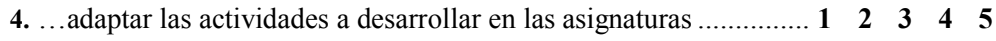

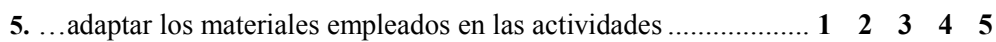

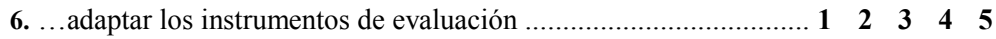

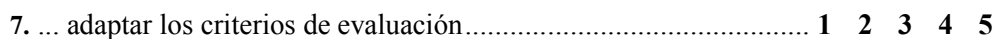

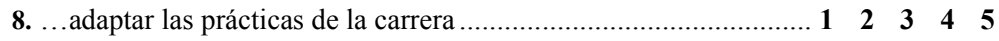

9. ... ampliar el tiempo para los exámenes y entrega de trabajos........ $1 \begin{array}{lllll}\mathbf{2} & \mathbf{3} & \mathbf{4} & \mathbf{5}\end{array}$

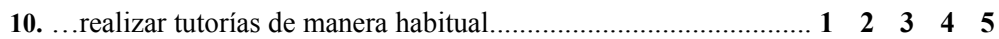




\section{CUNIDIS-d}

\section{Acción docente}

En la realidad del aula el profesorado:

\begin{tabular}{|c|c|c|}
\hline adapta los objetivos de las asignaturas ..................................... 1 & 2 & 3 \\
\hline enidos las asignaturas ...................................... 1 & 2 & 3 \\
\hline las clases............................ 1 & 2 & 3 \\
\hline 4... adapta las actividades a desarrollar en las asignaturas ................ 1 & 2 & 3 \\
\hline las actividades ...................... 1 & 2 & 3 \\
\hline . 1 & 2 & J \\
\hline ción ............................................ 1 & 2 & 5 \\
\hline ra & 2 & 5 \\
\hline ‥ & 2 & $J$ \\
\hline & & \\
\hline
\end{tabular}

\section{Accesibilidad}

Respecto a la accesibilidad:

21...la Facultad/Escuela no tiene barreras arquitectónicas . $\begin{array}{lllll}1 & 2 & 3 & 4 & 5\end{array}$

22...el equipamiento de las clases está adaptado $\begin{array}{lllll}1 & 2 & 3 & 4 & 5\end{array}$

23...las condiciones de las clases favorecen el acceso y movilidad .... $1 \begin{array}{lllll}1 & 2 & 3 & 4 & 5\end{array}$

24...la disposición de las clases permite el trabajo en grupo...... $\begin{array}{lllll}1 & 2 & 3 & 4 & 5\end{array}$

25...se emplean tecnologías para el seguimiento de las clases $\begin{array}{lllll}1 & 2 & 3 & 4 & 5\end{array}$

26...los materiales impresos/audiovisuales están adaptados $\begin{array}{lllll}1 & 2 & 3 & 4 & 5\end{array}$

27...cuentan con apoyos humanos/materiales para seguir las clases... $1 \begin{array}{lllll}1 & 2 & 3 & 4 & 5\end{array}$

28...realizan todas las prácticas de la carrera. $\begin{array}{lllll}1 & 2 & 3 & 4 & 5\end{array}$

29...participan en todas las actividades de clase $\begin{array}{lllll}1 & 2 & 3 & 4 & 5\end{array}$

30...tienen similares dificultades que el resto de los compañeros/as... $1 \begin{array}{lllll}1 & 2 & 3 & 4 & 5\end{array}$

\section{Comunidad Universitaria}

Respecto a la sensibilización y relaciones de los estudiantes con discapacidad:

31. Las campañas de la Universidad para la sensibilización y concienciación sobre discapacidad son adecuadas

32. Todos los estudiantes pueden participar en las actividades culturales, deportivas y de ocio que organiza la Universidad......... $1 \begin{array}{lllll}1 & 2 & 3 & 4 & 5\end{array}$

33. Los compañeros respetan la disposición de la clase para facilitar el acceso y movilidad de estos estudiantes

34. La comunicación con el profesorado es fluida.

35. La relación de los estudiantes y el profesorado de la Facultad/Escuela es adecuada 
CUNIDIS-d

36. La relación de los estudiantes y el resto de compañeros de la Facultad/Escuela es la adecuada.....

37. El profesorado de la Universidad está formado para dar respuesta a los estudiantes con discapacidad... $\begin{array}{lllll}1 & 2 & 3 & 4 & 5\end{array}$

38. Un estudiante con discapacidad puede estudiar cualquier carrera. $\begin{array}{lllll}1 & 2 & 3 & 4 & 5\end{array}$

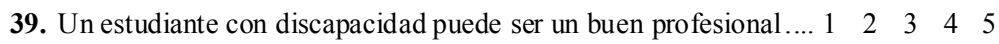

40. La Universidad está preparada para formar y atender a estudiantes con discapacidad. $\begin{array}{lllll}1 & 2 & 3 & 4 & 5\end{array}$

41. ¿Qué otras acciones podría realizar la Universidad para favorecer la inclusión de los estudiantes con discapacidad?

42. ¿Qué acciones consideras que puedes hacer como estudiante?

43. ¿Deseas realizar alguna otra aportación, valoración u opinión? 